\title{
The advances in immunotherapy for hepatocellular carcinoma
}

\author{
Fan Zhang ${ }^{1,2}$, Yumin $\mathrm{Li}^{1,2}$ \\ 'Department of Oncology Surgery, Lanzhou University Second Hospital, Lanzhou 730030, China. \\ ${ }^{2}$ Key Laboratory of Digestive System Tumors of Gansu Province, Lanzhou University Second Hospital, Lanzhou 730030, Gansu, \\ China. \\ Correspondence to: Dr. Yumin Li, Key Laboratory of Digestive System Tumors of Gansu Province, Lanzhou University Second \\ Hospital, 82 Cuiyingmen, Lanzhou 730030, Gansu, China. E-mail: liym@lzu.edu.cn
}

\begin{abstract}
How to cite this article: Zhang F, Li Y. The advances in immunotherapy for hepatocellular carcinoma. Hepatoma Res 2020;6:40. http://dx.doi.org/10.20517/2394-5079.2020.20
\end{abstract}

Received: 1 Mar 2020 First Decision: 26 Apr 2020 Revised: 25 May 2020 Accepted: 8 Jun 2020 Published: 10 Jul 2020

Academic Editor: Ying-Hong Shi Copy Editor: Cai-Hong Wang Production Editor: Tian Zhang

\begin{abstract}
Hepatocellular carcinoma (HCC) is one of the malignant tumors with higher incidence and mortality worldwide. Recently, significant progress has been made in uncovering immunotherapy in HCC, for instance programmed death-1, cytotoxic T-lymphocyte antigen 4, chimeric antigen receptor T-cell therapy, T cell receptor $\mathrm{T}$ cell therapy, dendritic cell vaccine, and cytokine-induced killer cells. This paper reviews the advances in immunotherapy and focuses on the results of many of preclinical studies and clinical trials in the field, as well as some of the promising therapeutic strategies for $\mathrm{HCC}$ in the future.
\end{abstract}

Keywords: HCC, PD-1, PD-L1, CTLA-4, CAR-T, T cell receptor, DC, CIK

\section{INTRODUCTION}

Hepatocellular carcinoma (HCC) was predicted to have the sixth highest incidence and the second highest mortality of malignant tumors worldwide in $2018^{[1]}$. The risk factors for HCC are closely related with lifestyle, chronic hepatitis B virus (HBV) and hepatitis C virus (HCV) infection, fatty liver disease, and cirrhosis ${ }^{[2-4]}$. The management of HCC involves a multidisciplinary team approach, considering not only the tumor stage and patient complications but also the seriousness of damaged liver function, as most HCC treatments can aggravate the severity of disease ${ }^{[5]}$. Although surgical resection remains the cornerstone of HCC therapy, limitations are caused by high recurrence rates after surgery because HCC is often diagnosed at advanced stage $^{[6]}$. Liver transplantation (LT) is the optimal treatment means for early-stage HCC, but limitations of LT are caused by organ shortage, tumor recurrences, and low-ratio eligibility. Comprehensive therapies for advanced HCC patients, such as radiotherapy, chemotherapy, interventional therapy, and targeted therapy, have been developed, but the 5 -year survival rate remains low ${ }^{[7]}$.

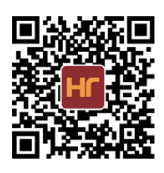


Cancer immunotherapy was selected as its annual breakthrough in Science journal in 2013. Following the advancements of immunotherapy in solid tumors over the last few years, as shown by the results of immune checkpoint inhibitors (ICIs) in lung cancer, renal cell cancer and melanoma ${ }^{[8]}$, in recent years, ICIs with antiCTLA-4 antibodies and anti-PD-1/PD-1L antibodies have been utilized to treat advanced melanoma ${ }^{[9]}$. In 2018, because of the achievements in the treatment of cancer with ICIs of CTLA-4 and PD-1/PD-L1, James P. Allison and Tasuku Honjo were awarded the Nobel prize.

Chimeric antigen receptor T-cell (CAR-T) immunotherapy has become more popular in the last decade as an antitumor therapy. Anti-CD19 CAR-T cell was approved by the FDA for treatment of subjects up to 25 years of age with B-cell acute lymphoblastic leukemia in $2017^{[10]}$. This article mainly summarizes the advances in ICIs and cellular immunotherapy for HCC.

\section{PD-1/PD-L1}

PD-1 is expressed on a subset of thymocytes and is upregulated on activated T cell, B cell, and myeloid cells $^{[11]}$. Two ligands for PD-1 were identified in 2000 and 2001 and named PD-L1 and programmed death ligand 2 (PD-L2), respectively ${ }^{[1,13]}$. PD-L1 is mainly expressed on stationary T cells, B cells, DC, and hepatoma cells, while PD-L2 is only expressed on DC and macrophages ${ }^{[14-16]}$. In theory, the interaction of PD-1 and PD-L1 expressed on immature T cells can interfere with activation. Similarly, if PD-L1 is highly expressed on tumor cell, the ligand receptor interactions between tumor cells and activated $\mathrm{T}$ cells triggers the immunosuppressive response, leading to immune tolerance ${ }^{[17]}$. It provides a theoretical basis for the treatment of PD-L1 in HCC.

It has been demonstrated that PD-L1 is overexpressed in HCC tissues; however, the results are controversial with respect to $\mathrm{PD}-\mathrm{L} 1$ as predictive biomarkers for $\mathrm{HCC}^{[18]}$. Several studies have reported that the higher PD-L1 expression on tumor cell in HCC patients were related with worse prognosis and tumor recurrence, and the studies also showed PD-L1 expression on macrophages was associated with favorable survival rate $^{[19-23]}$. However, two studies suggested that the expression of PD-L1 was not significantly correlated with survival outcomes in $\mathrm{HCC}^{[24,25]}$. Both soluble PD-1 (sPD-1) and soluble PD-L1 (sPD-L1) were prognostic factors with opposite prognostic values for HCC patients, while sPD-1 and sPD-L1 were not significantly related with PD-L1 expression in tumor ${ }^{[26]}$. However, two studies suggested plasma SPD-1 was associated with HBV activity and increased risk of HCC $^{[27,28]}$.

Liang et al. ${ }^{[29]}$ illustrated that inhibition of PD-1 can suppress the growth of hepatoma and promote the apoptosis of hepatoma. Increased expression levels of PD-1 were detected in peripheral blood and tumor infiltrating lymphocytes (TILs) of recurrent HCC patients ${ }^{[30]}$. Blockade of PD-1 on TILs can restore antitumor effects of TILs ${ }^{[31]}$. However, sPD-1/sPD-L1 was not associated with either PD-L1 expression of tumor cell or the numbers of CD4-positive TILs and CD8-positive TILs ${ }^{[26]}$. Tumor infiltrating neutrophils as a new target of immunotherapy participate in tumor progression, while the tumor microenvironment (TME) induces impaired antitumor immunity via the modulation of PD-L1 expression on tumor infiltrating neutrophils ${ }^{[32]}$. PD-L1 was positively associated with expression of $\mathrm{CD} 3$ and $\mathrm{CD} 8$ in HCC samples ${ }^{[23]}$. PD-L1 expression on macrophages is also a prognostic factor for HCC patients, and it could activate high levels of CD8(+) cytotoxic T-lymphocyte (CTL) infiltration and immune related gene expression ${ }^{[21]}$.

Since immune checkpoint molecules are recognized as vital indicators of HCC progress, series of clinical trials with ICIs have been implemented to confirm their potential function for advanced HCC. Nivolumab was approved by the FDA as immunotherapy for advanced stage HCC in $2017^{[33]}$. The efficiency of nivolumab was observed in a Phase I/II non-comparative trial (CheckMate 040) of patients with HCC and prior sorafenib treatment ${ }^{[33]}$. Forty-eight patients were treated with nivolumab in a dose-escalation phase. Then, since nivolumab showed adequate safety and feasibility, 214 patients from 39 sites in 11 countries received 
nivolumab in a dose-expansion phase. The objective response rates (ORR) of nivolumab were $15 \%$ in the dose-escalation phase and $20 \%$ in the dose-expansion phase, suggesting that efficacy of nivolumab is not efficient. Twelve of 48 patients had Grade 3/4 treatment-associated adverse events. Although the study was positive in favor of anti-PD-1 treatment, it is worth corroborating the efficacy of nivolumab in a therapeutic schedule. Pre-treatment of sorafenib might potentiate the therapeutic response to subsequent treatment with nivolumab. In real-life experience from three German centers, Grade 3 treatment-associated events occurred in two patients (5.9\%), and the partial response rate and stable disease rate in 34 patients with advanced HCC and nivolumab treatment were $11.8 \%$ and $23.5 \%$ in line with data from the CheckMate 040 trial ${ }^{[34]}$.

Pembrolizumab is also an antibody against PD-1. In a Phase II open-label non-randomized trial (KEYNOTE-224) to assess the efficacy of pembrolizumab as an alternative second-line treatment for HCC patients, the median overall survival (OS) was 12.9 months with a disease control rate of $61 \%$ and ORR of $17 \%{ }^{[35]}$. Grade 3 toxicities arose in $25(24 \%)$ of the 104 patients. Hence, pembrolizumab is temporarily approved by the FDA as a second-line therapy for advanced HCC, but it still needs to be verified by the results of more Phase III trials ${ }^{[36]}$. A Phase III randomized, double-blind trial to further assess the efficacy of pembrolizumab versus placebo in HCC patient is still ongoing (NCT02702401). A Phase II study evaluating camrelizumab for HCC patients with resistance to systemic treatment displayed ORR of $13.8 \%$ and acceptable treatment-related adverse events in Chinese advanced HCC patients ${ }^{[37]}$. In addition to monotherapy, possible multimodality therapeutic options involving ICIs are under investigation. Some research has observed that ICIs of PD-L1 in combination with sorafenib, lenvatinib, rapamycin, and histone deacetylase inhibitor may enhance therapeutic benefit ${ }^{[38-41]}$.

Clinical trials of PD-1 antibodies combined with other adjuvant therapy, e.g., transarterial chemoembolization (TACE) and selective internal radiation treatment, are currently in progress. In addition, different combination regimens, which depend on understanding of the actual immune mechanisms in the various combinations, could help us select the optimal therapeutic option for advanced HCC.

\section{CTLA-4}

CTLA-4 downregulates activation of T cells by interacting with CD80/CD86 on the surface of DCs ${ }^{[42]}$. For naive $\mathrm{T}$ cell activation, $\mathrm{CD} 28$ on $\mathrm{T}$ cells provides the second activation signal by binding to $\mathrm{CD} 80 / \mathrm{CD} 86$ on $\mathrm{DCs}^{[43]}$. CTLA-4 has a greater affinity for interacting with CD80/CD86 than CD28 so that it interferes in T cell activation ${ }^{[4]}$. Various single nucleotide polymorphisms (SNP) in CTLA-4 have been well-studied. Several studies observed that polymorphism of CTLA- 4 was associated with increased susceptibility to HCC and haplotypes of CTLA-4 may affect the risk of $\mathrm{HCC}^{[45-48]}$.

In 2013, the first CTLA-4 blocking inhibitor in practical HCC treatment was tremelimumab, which displayed promising antitumor activity and acceptable safety ${ }^{[49]}$. In a clinical trial to validate efficacy of tremelimumab in patients with HCC and HCV infection, partial response rate and disease control rate were $17.6 \%$ and $76.4 \%$, respectively ${ }^{[49]}$. Duffy et al.$^{[50]}$ attempted to combine tremelimumab with ablation as an expected therapeutic option for patients with advanced HCC (NCT01853618). Five partial responses were observed in 19 patients, with median OS of 12.3 months. Tremelimumab is a human IgG2 monoclonal antibody that blocks the binding of CTLA-4 on the surface of activated $\mathrm{T}$ cell ${ }^{[51]}$. It has been reported that tremelimumab could induce tumor responses in a subset of patients with non-small cell lung cancer and refractory biliary tract cancer ${ }^{[52,53]}$. Tremelimumab therapy could elevate the amount of $\mathrm{T}$ cells in the peripheral blood and TILs, and CD4(+) PD-1(+) cells were more likely to be activated by tremelimumab ${ }^{[54]}$. An important adverse effect of tremelimumab is transaminitis, as a high proportion of reversible Grade 3/4 transaminitis was observed in both the above-mentioned studies.

Preclinical data based on series of solid tumors indicate that dual immune checkpoint blockade is synergistic and leads to higher response rates and improved treatment outcomes compared to monotherapy. Most 
clinical data suggest that both CTLA-4 and PD-1/PD-L1 blockade a portion of HCC patients. Compared to CTLA-4 blockade, PD-1 and PD-L1 blockade showed relatively higher ORR, which could reach 10\%-20\% in advanced HCC patients. PD-1/PD-L1 blockade agents were more tolerable and less hepatoxic. Further studies for combined PD-1/PD-L1 and CTLA-4 blockades in HCC treatment are still expected, which may help to mitigate the adverse effects of the treatment. Immune checkpoint blockade in advanced HCC combined with other conventional ablative treatments, such as radiofrequency ablation (RFA) or microwave, TACE, chemotherapy, targeted medicine, or surgery would be the most promising approach for HCC patients. However, for unresectable advanced HCC, it is more appropriate to search for other combination strategies, such as the combination with multi-kinase inhibitors, vaccines, and oncolytic viruses, as well as dual inhibition of two immune checkpoint molecules.

Based on current evidence, combination therapies with CTLA-4 are now an expected direction for the immunotherapy of advanced HCC patients in the future. A Phase III study (NCT03298451) of durvalumab with or without tremelimumab vs. sorafenib in patients of advanced HCC enrolled about 1,350 patients and explored two treatment schedules. Given the limited data to date, further testing of this combination is ongoing in a Phase II expansion. Most ongoing clinical trials have been designed to assess the efficiency of the combination strategies.

\section{CAR-T CELL THERAPY}

CD19 targeted CAR-T immunotherapy is an expecting therapeutic option that has shown high efficacy in treating hematologic malignancies ${ }^{[55]}$. Moreover, a great number of CAR-T cell products in solid tumors has also been investigated in preclinical and clinical studies. In 2008, Wilkie et al. ${ }^{[56]}$ reported for the first time that MUC1 targeted CAR-T could significant delay tumor growth in solid tumor ${ }^{[56]}$. The basic principle of CAR-T cell therapy is the modification of $\mathrm{T}$ cells with CARs, so that they can identify tumor cells, and then the retransfusion of these CAR-T cells into the human body to fight against the target cells ${ }^{[57,58]}$. Several studies have found that GPC3-targeted CAR-T cell therapy can eliminate HCC cells in preclinical research ${ }^{[59-61]}$. GPC3 is a 70-kDa heparan oncofetal proteoglycans that is located on the tumor cell membrane ${ }^{[62]}$. It has been demonstrated that GPC3 is detected in HCC tissues with higher expression but not in normal tissues ${ }^{[63]}$. A Phase I trial (NCT02395250) of 13 Chinese GPC3-positive HCC patients illustrated the safety and preliminary efficacy of GPC3 CAR-T cells in $2017^{[64]}$. According to the patient's tolerance, the preliminary analysis showed that GPC3 targeted CAR-T combined with the lymphodepleting conditioning had a certain efficacy ${ }^{[64]}$. The pre-clinical studies for dual-targeted CAR-T cells co-expressing GPC3 CARs and GPC3-specific CAR-modified T cells fusing a soluble PD1-CH3 fusion protein showed promising results $^{[60,61]}$.

$\alpha$-fetoprotein (AFP) has been used not only as a biomarker for surveillance and diagnosis of HCC, but also as a target for immunotherapy ${ }^{[65]}$. In a clinical trial of 15 HCC patients who were given a subcutaneous injection of AFP-derived peptides, 1 patient had a complete response and the disease stabilized in 8 patients ${ }^{[66]}$. AFP, an intracellular/secreted protein, can generate AFP peptide-major histocompatibility complex (MHC) complexes as targets for CAR T-cell therapy for solid tumors. Liu et al. ${ }^{[67]}$ detected that AFPtargeted CAR-T cells showed significant antitumor capacity in a mouse model Additionally, AFP-derived vaccines can augment the activity of ICIs, leading to deterioration of HCC.

The experience from successful clinical studies of hematologic malignancies provides us with the understanding that, although selection of the specific antigen to avoid off-target or on-target/off-tumor toxicity is a primary task to be tackled, for HCC, the challenge of CAR-T is the need to ascertain a specific neoantigen and overcome the TME, gut microbiome, and HCC genomic features. Furthermore, the activation, proliferation, and persistence of CAR-T are more important for therapy. In addition, standardization in the production of CAR-T and achieving individualized treatment should be considered. 


\section{TCR-T CELL THERAPY}

TCR-T cell immunotherapy, as one of the novel and effective antitumor treatment means, has been widely studied in oncotherapy. In 2011, Parkhurst et al. ${ }^{[68]}$ firstly reported that human carcinoembryonic antigen (CEA)-targeted TCR-T cell therapy could induce objective regression of metastatic colorectal cancer ${ }^{[68]}$. The mechanisms of TCR-T cell therapies are similar to CAR-T immunotherapy. TCR-T therapy also modifies the autologous T cells with TCR, and then retransfusion expands TCR-T cell back into the patient to recognize and eliminate tumor cell, but the mechanisms for identifying antigens are quite different from CAR-T cell therapies ${ }^{[57]}$. The specific antigens recognized by CAR-T cell are all cell membrane antigens, while TCR-T cell can identify intracellular and cell membrane antigen peptides presented by MHC molecules ${ }^{[69]}$. In HBVrelated HCC, by performing the high-throughput TCR sequence of TILs in tumor and matched adjacent normal tissues, Lin et al ${ }^{[70]}$ found that the combination of TCR repertoire overlap and TNM stage showed a better prognostic effect for HCC than TNM stage. Qasim et al. ${ }^{[71]}$ firstly reported an HBV-related end-stage HCC case treated with HBV surface antigen as a target for HBV-specific TCR T cell therapy in 2015. In most HBV-related HCC, HBV integrations have been observed and can result in the expression of HCC cells ${ }^{[72]}$. HCC cells comprise fragments of integrated HBV-DNA that encodes peptides, which can be identified by T cells ${ }^{[73]}$. Another trial was conducted in two advanced HCCs patients who underwent liver transplantation with HCC relapses ${ }^{[74]}$. During the one-year period of follow up, the volume of 5/6 pulmonary metastases was decreased in one patient receiving HBV-specific TCR T cell therapy ${ }^{[74]}$. Basic studies of TCR-T cells therapy with specific targets, such as HCV, AFP, and GPC, may be a promising immunotherapy strategy for HCC in the future ${ }^{[75-77]}$. With TCR-T immunotherapy, the efficacy and side effects seem to mainly depend on the quality of the specific target and the TCR structure. The primary challenge is the discovery of new targets, particularly in the promising field of neoantigens. However, it should be emphasized that neoantigens may be expressed on a subset of tumor cells due to heterogeneity of tumor cell; otherwise, it may cause immune escape.

\section{DENDRITIC CELL VACCINE}

DCs are powerful antigen-presenting cells that can stimulate $\mathrm{T}$ cells to induce antitumor activity. The infiltration of DCs in tumor tissue was closely associated to the improved clinical prognosis in HCC patients ${ }^{[78,79]}$. In 2002, Ladhams et al ${ }^{[80]}$ firstly reported two patients with end-stage HCC treated with autologous DCs vaccination co-cultured with autologous HCC antigens. The efficacy of DC vaccination loaded with tumor antigens from different sources has been investigated in clinical studies. Lee et al. ${ }^{[81]}$ reported a trial which enrolled 31 advanced HCC patients receiving DC vaccine pulsed with autologous tumor lysates in 2005. They reported that rates of partial response and stable disease were $12.9 \%$ and $54.8 \%$, respectively. A Phase II clinical trial reported disease control rate was $28 \%$ for advanced HCC patients with DC vaccination pulsed HepG2 lysate ${ }^{[82]}$. In another study of note, El Ansary et al ${ }^{[83]}$, also using DC vaccine pulsed with HepG2 lysate, showed that DC vaccination could partially improve survival outcome. DC vaccination loaded with autologous tumor lysates or ex vivo HepG2 cell lysate were feasible and effective.

However, the efficacy of DCs vaccination pulsed with tumor cell lysate is not satisfactory, and thus the use of specific antigen-modified DC vaccination has been attempted. Kakumu et al. ${ }^{[84]}$ suggested that the depressed function of DCs is associated with pathogenesis of HCC with HBV or HCV infection. Several preclinical studies indicated that DCs infected with AFP or HBV antigen or both were effective strategies to enhance efficacy of DC-based vaccine ${ }^{[85-87]}$. GPC3-modified DCs were potent in inducing T cell proliferation and interferon (IFN)-y production ${ }^{[88]}$. Tada et al. ${ }^{[89]}$ reported a clinical effect was observed in one of the five patients receiving DC vaccination pulsed with AFP, GPC3, and MAGE-1 fusion proteins in 2012. Subsequently, a large sample study confirmed that the median time of progression of HCC patients with DC vaccination pulsed with AFP, GPC3 and MAGE-1 fusion proteins was longer than the control group (36.6 months vs. 11.8 months) ${ }^{[90]}$. 
DCs pulsed with Hsp70 peptide and OK-432 can enhance efficacy of vaccine inducing $\mathrm{T}$ cell proliferation and CTL response ${ }^{[91,92]}$. In a clinical trial using Hsp70-DC vaccination, 2/12 patients demonstrated complete response and $5 / 12$ patients demonstrated stable diseas ${ }^{[93]}$. In our previous meta-analysis, we concluded DCbased therapy could prolong the median progression free survival (PFS) time and median OS time ${ }^{[94]}$.

However, the maturation of DC was closely associated with efficacy of DC immunotherapy. The stimulatory capacity of dendritic cells from HCC patients was significantly lower than dendritic cells from liver cirrhosis tissue and normal samples ${ }^{[95]}$. Meanwhile, the numbers of CD83-positive DCs in HCC specimens were significantly lower compared with liver cirrhosis samples ${ }^{[96]}$. Therefore, it is very important to improve the maturation of DC, increase antigen source, and depress TME. Various stimuli, such as tumor necrosis factor alpha, lipopolysaccharide, IFN gamma, CD40-ligand, PEG10, IL-12, EpCAM, and HCA661, can significantly increase the stimulatory capacity of DCs ${ }^{[97-102]}$. Tumor endothelial marker 8 modified DCs could stimulate antitumor immunity by disrupting tumor vasculature, and DCs loaded with specific peptide, such as FoxM1, could significantly inhibit tumor growth and metastasis ${ }^{[103,104]}$. In addition, RFA can create an antigenic source with stimuli appropriate for maturation of $\mathrm{DCs}^{\left[{ }^{[105]}\right.}$. Regulatory $\mathrm{T}$ cells, producing immunosuppressive cytokine IL-10, were concentrated within HCC tissue and were inducted by local TME to interfere the differentiation and maturation of $\mathrm{DC}^{[7]}$. To overcome the immunosuppressive TME, Hu et al. ${ }^{[106]}$ introduced a promising vaccine candidate, which combine the DC/tumor cell fusion vaccine with nanoparticles of folate-modified chitosan carrying interferon-induced protein-10, which could effectively inhibit tumor cell proliferation and significantly reduce myeloid-derived suppressor cells in mouse immune organs.

\section{CIK/DC-CIK}

CIK are a subset of non-MHC-restricted T lymphocytes with immune modulatory effects and a crucial role in anti-tumor immunotherapy ${ }^{[107]}$. Several studies suggested that CIK cells co-cultured with DCs can significantly enhance antitumor efficiency ${ }^{[108,109]}$. Qiu et al.$^{[10]}$ reported that alpha-Gal epitope-pulsed DCCIK therapy remarkably prolonged the survival of patients with stage III primary HCC as compared to the controls (17.1 months vs. 10.1 months). In a retrospective study from 45 patients with metastatic HCC, median OS of DC-CIK immunotherapy plus ablation (32 months) or ablation (17.5 months) was higher than untreated group (3 months) ${ }^{[111]}$. In a propensity score-matched analysis, autologous CIK immunotherapy showed significantly longer RFS than the control group ${ }^{[112]}$. After 5 -year follow-up, CIK immunotherapy show a significant reduction in the risk of recurrence or death ${ }^{[113]}$. The combination therapies DC-CIK with other therapeutic options, such as TACE, could improve the antitumor efficacy. Guo et al. ${ }^{[114]}$ reported that DC-CIK therapy combined with TACE can improve the PFS but not the OS outcomes. However, TACE plus DC-CIK therapy for HCC patients is superior to TACE alone in improving median OS and PFS in a metaanalysis $^{[115]}$. Zhou et al. ${ }^{[116]}$ analyzed that clinical benefit rate of sorafenib combined with DC-CIK is higher than oral administration of sorafenib $(88.6 \%$ vs. $41.9 \%)$ in a meta-analysis.

To enhance the therapeutic efficacy of CIK cells, several pre-clinical studies suggested that co-culture of modified DCs, such as IL24-modified DCs, AFP-modified DCs, and GPC3-modified DCs, with CIKs can significantly promote CIKs differentiation and enhance lytic activity of CIK cells ${ }^{[117-119]}$. They provided a promising DC-CIK vaccine candidate for further clinical trials of HCC patients. Indeed, CIK or DC-CIK immunotherapies from autologous or allogeneic donors have already been extensively used in solid tumor patients. In our clinical center, we have experience with more than 100 gastric cancer patients with DC-CIK immunotherapy and have found that treatment outcomes were effective, safe, and feasible for gastric cancer patients. The standardization in the preparation and criteria of indication are progressing; several clinical trials are registered and ongoing. CIK or DC-CIK immunotherapies, combined with other antitumor agents, should be considered. 


\section{CONCLUSION AND OUTLOOK}

Immunotherapies appear to be a promising treatment for advanced HCC. Multiple prospective studies are attempting to validate the therapy outcomes with PD-1/PD-L1 and/or CTLA-4 blockade. However, only a small proportion of HCC patients effectively respond to immunotherapies and much research is still needed. One of the future directions for immunotherapies is combination therapies with other ICIs, TKIs, vaccines, and oncolytic viruses, as well as conventional treatments in various stages of patients to improve the antitumor efficacy. In addition, it is the important to research how to elevate immunotherapy efficacy and ascertain the biomarkers of predictive therapeutic response to immunotherapy. For example, TMB has been used in several tumor types to predict therapeutic response to anti-PD-1 therapy. In the future, we expect to identify more predictive biomarker subsets which can be used to accurately evaluate the efficacy of immunotherapy.

CAR-T technology and its application has been hailed as a scientific breakthrough in the field of hematological tumors. Application of CAR-T therapy and TCR to treat HCC is expected to be a promising therapeutic method. The crucial challenge is the need to identify specific antigens; overcome the TME, gut microbiome, and HCC genomic features; and guard against adverse effects. Furthermore, the activation, proliferation, and persistence of CAR-T immunotherapy should be considered with the outcomes of treatments for HCC. In addition, standardization in the production of CAR-T and achieving individualized treatment should be considered.

Different modifications of DC vaccine or DC-CIK therapy, such as selection of specific antigen targets and appropriate immunologic adjuvant, may elevate the effectiveness and safety in further studies. Dendritic cells lead to an increase in the naturally occurring neoantigen-specific immune response as well as the diversity of neoantigens. The combination of DC vaccination with other immunotherapies, e.g., TCR-T, may be a novel treatment modality in the future.

Because of the heterogeneity of tumor cells and the complexity of immuno-regulatory mechanisms, multimodality therapies based on immunotherapy represent the next step in clinical antitumor efficacy, which will enable advancing the field and improving the outcomes of HCC patients.

\section{DECLARATIONS}

\section{Authors' contributions}

Planned and designed of the study: Li Y

Searched the literature and wrote the manuscript, performed revisions, read and approved the final manuscript for publication: Zhang F, Li Y

\section{Availability of data and materials}

Not applicable.

\section{Financial support and sponsorship}

The present study was supported by the National Natural Science Foundation of China (31770537), International S \& T Cooperation Program of China (ISTCP) (2015DFA31650); International S \& T Cooperation Program of Gansu Province (18YF1WA113).

\section{Conflicts of interest}

All authors declared that they have no conflicts of interest.

\section{Ethics approval and consent to participate}

Not applicable. 


\section{Consent for publication}

Not applicable.

\section{Copyright}

(c) The Author(s) 2020.

\section{REFERENCE}

1. Bray F, Ferlay J, Soerjomataram I, Siegel RL, Torre LA, et al. Global cancer statistics 2018: GLOBOCAN estimates of incidence and mortality worldwide for 36 cancers in 185 countries. CA Cancer J Clin 2018;68:394-424.

2. Tian Y, Yang B, Qiu W, Hao Y, Zhang Z, et al. ER-residential Nogo-B accelerates NAFLD-associated HCC mediated by metabolic reprogramming of oxLDL lipophagy. Nat Commun 2019;10:3391.

3. Papatheodoridis GV, Chan HL, Hansen BE, Janssen HL, Lampertico P. Risk of hepatocellular carcinoma in chronic hepatitis B: assessment and modification with current antiviral therapy. J Hepatol 2015;62:956-67.

4. Singal AG, Rich NE, Mehta N, Branch AD, Pillai A, et al. Direct-acting antiviral therapy for hepatitis C virus infection is associated with increased survival in patients with a history of hepatocellular carcinoma. Gastroenterology 2019;157:1253-63.e2.

5. Yang JD, Hainaut P, Gores GJ, Amadou A, Plymoth A, et al. A global view of hepatocellular carcinoma: trends, risk, prevention and management. Nat Rev Gastroenterol Hepatol 2019;16:589-604.

6. Desert R, Rohart F, Canal F, Sicard M, Desille M, et al. Human hepatocellular carcinomas with a periportal phenotype have the lowest potential for early recurrence after curative resection. Hepatology 2017;66:1502-18.

7. Bruix J, da Fonseca LG, Reig M. Insights into the success and failure of systemic therapy for hepatocellular carcinoma. Nat Rev Gastroenterol Hepatol 2019;16:617-30.

8. Topalian SL, Hodi FS, Brahmer JR, Gettinger SN, Smith DC, et al. Safety, activity, and immune correlates of anti-PD-1 antibody in cancer. N Engl J Med 2012;366:2443-54.

9. Mason R, Au L, Ingles Garces A, Larkin J. Current and emerging systemic therapies for cutaneous metastatic melanoma. Expert Opin Pharmacother 2019;20:1135-52.

10. Administration UFaD. FDA approves tisagenlecleucel for B-cell ALL and tocilizumab for cytokine release syndrome. 2017. Available from: https://www.fda.gov/Drugs/InformationOnDrugs/ApprovedDrugs/ucm606540.htm [Last accessed on 16 Jun 2020]

11. Agata Y, Kawasaki A, Nishimura H, Ishida Y, Tsubata T, et al. Expression of the PD-1 antigen on the surface of stimulated mouse T and B lymphocytes. Int Immunol 1996;8:765-72.

12. Latchman Y, Wood CR, Chernova T, Chaudhary D, Borde M, et al. PD-L2 is a second ligand for PD-1 and inhibits T cell activation. Nat Immunol 2001;2:261-8.

13. Freeman GJ, Long AJ, Iwai Y, Bourque K, Chernova T, et al. Engagement of the PD-1 immunoinhibitory receptor by a novel B7 family member leads to negative regulation of lymphocyte activation. J Exp Med 2000;192:1027-34.

14. Finkelmeier F, Waidmann O, Trojan J. Nivolumab for the treatment of hepatocellular carcinoma. Expert Rev Anticancer Ther 2018;18:1169-75.

15. Yarchoan M, Xing D, Luan L, Xu H, Sharma RB, et al. Characterization of the Immune Microenvironment in Hepatocellular Carcinoma. Clin Cancer Res 2017;23:7333-9.

16. Mocan T, Sparchez Z, Craciun R, Bora CN, Leucuta DC. Programmed cell death protein-1 (PD-1)/programmed death-ligand-1 (PD-L1) axis in hepatocellular carcinoma: prognostic and therapeutic perspectives. Clin Transl Oncol 2019;21:702-12.

17. Shi F, Shi M, Zeng Z, Qi RZ, Liu ZW, et al. PD-1 and PD-L1 upregulation promotes CD8(+) T-cell apoptosis and postoperative recurrence in hepatocellular carcinoma patients. Int J Cancer 2011;128:887-96.

18. Long J, Qu T, Pan XF, Tang X, Wan HH, et al. Expression of programmed death ligand-1 and programmed death 1 in hepatocellular carcinoma and its clinical significance. J Cancer Res Ther 2018;14:S1188-92.

19. Ma LJ, Feng FL, Dong LQ, Zhang Z, Duan M, et al. Clinical significance of PD-1/PD-Ls gene amplification and overexpression in patients with hepatocellular carcinoma. Theranostics 2018;8:5690-702.

20. Jung HI, Jeong D, Ji S, Ahn TS, Bae SH, et al. Overexpression of PD-L1 and PD-L2 is associated with poor prognosis in patients with hepatocellular carcinoma. Cancer Res Treat 2017;49:246-54

21. Liu CQ, Xu J, Zhou ZG, Jin LL, Yu XJ, et al. Expression patterns of programmed death ligand 1 correlate with different microenvironments and patient prognosis in hepatocellular carcinoma. Br J Cancer 2018;119:80-8.

22. Chang H, Jung W, Kim A, Kim HK, Kim WB, et al. Expression and prognostic significance of programmed death protein 1 and programmed death ligand-1, and cytotoxic T lymphocyte-associated molecule-4 in hepatocellular carcinoma. Apmis 2017;125:690-8.

23. Gabrielson A, Wu Y, Wang H, Jiang J, Kallakury B, et al. Intratumoral CD3 and CD8 T-cell densities associated with relapse-free survival in HCC. Cancer Immunol Res 2016;4:419-30.

24. Huang CY, Wang Y, Luo GY, Han F, Li YQ, et al. Relationship between PD-L1 expression and CD8+ T-cell immune responses in hepatocellular carcinoma. J Immunother 2017;40:323-33.

25. Umemoto Y, Okano S, Matsumoto Y, Nakagawara H, Matono R, et al. Prognostic impact of programmed cell death 1 ligand 1 expression in human leukocyte antigen class I-positive hepatocellular carcinoma after curative hepatectomy. J Gastroenterol 2015;50:65-75.

26. Chang B, Huang T, Wei H, Shen L, Zhu D, et al. The correlation and prognostic value of serum levels of soluble programmed death 
protein 1 (sPD-1) and soluble programmed death-ligand 1 (sPD-L1) in patients with hepatocellular carcinoma. Cancer Immunol Immunother 2019;68:353-63.

27. Cheng HY, Kang PJ, Chuang YH, Wang YH, Jan MC, et al. Circulating programmed death-1 as a marker for sustained high hepatitis B viral load and risk of hepatocellular carcinoma. PLoS One 2014;9:e95870.

28. Li N, Zhou Z, Li F, Sang J, Han Q, et al. Circulating soluble programmed death-1 levels may differentiate immune-tolerant phase from other phases and hepatocellular carcinoma from other clinical diseases in chronic hepatitis B virus infection. Oncotarget 2017;8:4602033.

29. Liang L, Ge K, Zhang F, Ge Y. The suppressive effect of co-inhibiting PD-1 and CTLA-4 expression on H22 hepatomas in mice. Cell Mol Biol Lett 2018;23:58.

30. Song B, Zhen S, Meng F. T cell inflammation profile after surgical resection may predict tumor recurrence in HBV-related hepatocellular carcinoma. Int Immunopharmacol 2016;41:35-41.

31. Liu F, Zeng G, Zhou S, He X, Sun N, et al. Blocking Tim-3 or/and PD-1 reverses dysfunction of tumor-infiltrating lymphocytes in HBVrelated hepatocellular carcinoma. Bull Cancer 2018;105:493-501.

32. He G, Zhang H, Zhou J, Wang B, Chen Y, et al. Peritumoural neutrophils negatively regulate adaptive immunity via the PD-L1/PD-1 signalling pathway in hepatocellular carcinoma. J Exp Clin Cancer Res 2015;34:141.

33. El-Khoueiry AB, Sangro B, Yau T, Crocenzi TS, Kudo M, et al. Nivolumab in patients with advanced hepatocellular carcinoma (CheckMate 040): an open-label, non-comparative, phase 1/2 dose escalation and expansion trial. Lancet 2017;389:2492-502.

34. Finkelmeier F, Czauderna C, Perkhofer L, Ettrich TJ, Trojan J, et al. Feasibility and safety of nivolumab in advanced hepatocellular carcinoma: real-life experience from three German centers. J Cancer Res Clin Oncol 2019;145:253-9.

35. Zhu AX, Finn RS, Edeline J, Cattan S, Ogasawara S, et al. Pembrolizumab in patients with advanced hepatocellular carcinoma previously treated with sorafenib (KEYNOTE-224): a non-randomised, open-label phase 2 trial. Lancet Oncol 2018;19:940-52.

36. Marquardt JU, Saborowski A, Czauderna C, Vogel A. The changing landscape of systemic treatment of advanced hepatocellular carcinoma: new targeted agents and immunotherapies. Target Oncol 2019;14:115-23.

37. Qin SK, Ren ZG, Meng ZQ, Chen ZD, Chai XL, et al. A randomized multicentered phase II study to evaluate SHR-1210 (PD-1 antibody) in subjects with advanced hepatocellular carcinoma (HCC) who failed or intolerable to prior systemic treatment. Ann Oncol 2018;29:viii719-20.

38. Llopiz D, Ruiz M, Villanueva L, Iglesias T, Silva L, et al. Enhanced anti-tumor efficacy of checkpoint inhibitors in combination with the histone deacetylase inhibitor Belinostat in a murine hepatocellular carcinoma model. Cancer Immunol Immunother 2019;68:379-93.

39. Li H, Li X, Liu S, Guo L, Zhang B, et al. Programmed cell death-1 (PD-1) checkpoint blockade in combination with a mammalian target of rapamycin inhibitor restrains hepatocellular carcinoma growth induced by hepatoma cell-intrinsic PD-1. Hepatology 2017;66:1920-33.

40. Chen ML, Yan BS, Lu WC, Chen MH, Yu SL, et al. Sorafenib relieves cell-intrinsic and cell-extrinsic inhibitions of effector T cells in tumor microenvironment to augment antitumor immunity. Int J Cancer 2014;134:319-31.

41. Kimura T, Kato Y, Ozawa Y, Kodama K, Ito J, et al. Immunomodulatory activity of lenvatinib contributes to antitumor activity in the Hepa1-6 hepatocellular carcinoma model. Cancer Sci 2018;109:3993-4002.

42. Butty V, Roy M, Sabeti P, Besse W, Benoist C, et al. Signatures of strong population differentiation shape extended haplotypes across the human CD28, CTLA4, and ICOS costimulatory genes. Proc Natl Acad Sci USA 2007;104:570-5.

43. Esensten JH, Helou YA, Chopra G, Weiss A, Bluestone JA. CD28 costimulation: from mechanism to therapy. Immunity 2016;44:973-88.

44. Valk E, Rudd CE, Schneider H. CTLA-4 trafficking and surface expression. Trends Immunol 2008;29:272-9.

45. Yang J, Liu J, Chen Y, Tang W, Liu C, et al. Association of CTLA-4 tagging polymorphisms and haplotypes with hepatocellular carcinoma risk: a case-control study. Medicine (Baltimore) 2019;98:e16266.

46. Wang C, Liu W, Zhao L, Dong Z. Association of cytotoxic T-lymphocyte antigen-4 + 49A/G gene polymorphism with hepatocellular carcinoma risk in Chinese. J Cancer Res Ther 2018;14:S1117-20.

47. Hu L, Liu J, Chen X, Zhang Y, Liu L, et al. CTLA-4 gene polymorphism +49 A/G contributes to genetic susceptibility to two infectionrelated cancers-hepatocellular carcinoma and cervical cancer. Hum Immunol 2010;71:888-91.

48. Gu X, Qi P, Zhou F, Ji Q, Wang H, et al. +49G > A polymorphism in the cytotoxic T-lymphocyte antigen-4 gene increases susceptibility to hepatitis B-related hepatocellular carcinoma in a male Chinese population. Hum Immunol 2010;71:83-7.

49. Sangro B, Gomez-Martin C, de la Mata M, Inarrairaegui M, Garralda E, et al. A clinical trial of CTLA-4 blockade with tremelimumab in patients with hepatocellular carcinoma and chronic hepatitis C. J Hepatol 2013;59:81-8.

50. Duffy AG, Ulahannan SV, Makorova-Rusher O, Rahma O, Wedemeyer H, et al. Tremelimumab in combination with ablation in patients with advanced hepatocellular carcinoma. J Hepatol 2017;66:545-51.

51. Ribas A, Camacho LH, Lopez-Berestein G, Pavlov D, Bulanhagui CA, et al. Antitumor activity in melanoma and anti-self responses in a phase I trial with the anti-cytotoxic T lymphocyte-associated antigen 4 monoclonal antibody CP-675,206. J Clin Oncol 2005;23:8968-77.

52. Antonia S, Goldberg SB, Balmanoukian A, Chaft JE, Sanborn RE, et al. Safety and antitumour activity of durvalumab plus tremelimumab in non-small cell lung cancer: a multicentre, phase 1b study. Lancet Oncol 2016;17:299-308.

53. Xie C, Duffy AG, Mabry-Hrones D, Wood B, Levy E, et al. Tremelimumab in combination with microwave ablation in patients with refractory biliary tract cancer. Hepatology 2019;69:2048-60.

54. Agdashian D, ElGindi M, Xie C, Sandhu M, Pratt D, et al. The effect of anti-CTLA4 treatment on peripheral and intra-tumoral T cells in patients with hepatocellular carcinoma. Cancer Immunol Immunother 2019;68:599-608.

55. Mohty M, Gautier J, Malard F, Aljurf M, Bazarbachi A, et al. CD19 chimeric antigen receptor-T cells in B-cell leukemia and lymphoma: current status and perspectives. Leukemia 2019;33:2767-78. 
56. Wilkie S, Picco G, Foster J, Davies DM, Julien S, et al. Retargeting of human T cells to tumor-associated MUC1: the evolution of a chimeric antigen receptor. J Immunol 2008;180:4901-9.

57. Zhao L, Cao YJ. Engineered T cell therapy for cancer in the clinic. Front Immunol 2019;10:2250.

58. Sadelain M, Riviere I, Riddell S. Therapeutic T cell engineering. Nature 2017;545:423-31.

59. Gao H, Li K, Tu H, Pan X, Jiang H, et al. Development of T cells redirected to glypican-3 for the treatment of hepatocellular carcinoma. Clin Cancer Res 2014;20:6418-28.

60. Chen C, Li K, Jiang H, Song F, Gao H, et al. Development of T cells carrying two complementary chimeric antigen receptors against glypican-3 and asialoglycoprotein receptor 1 for the treatment of hepatocellular carcinoma. Cancer Immunol Immunother 2017;66:47589.

61. Pan Z, Di S, Shi B, Jiang H, Shi Z, et al. Increased antitumor activities of glypican-3-specific chimeric antigen receptor-modified T cells by coexpression of a soluble PD1-CH3 fusion protein. Cancer Immunol Immunother 2018;67:1621-34.

62. Zhou F, Shang W, Yu X, Tian J. Glypican-3: a promising biomarker for hepatocellular carcinoma diagnosis and treatment. Med Res Rev 2018;38:741-67.

63. Baumhoer D, Tornillo L, Stadlmann S, Roncalli M, Diamantis EK, et al. Glypican 3 expression in human nonneoplastic, preneoplastic, and neoplastic tissues: a tissue microarray analysis of 4,387 tissue samples. Am J Clin Pathol 2008;129:899-906.

64. Zhai B, Shi D, Gao H, Qi X, Jiang H, et al. A phase I study of anti-GPC3 chimeric antigen receptor modified T cells (GPC3 CAR-T) in Chinese patients with refractory or relapsed GPC3 + hepatocellular carcinoma (r/r GPC3 + HCC). J Clin Oncol 2017;35:3049.

65. He Y, Hong Y, Mizejewski GJ. Engineering $\alpha$-fetoprotein-based gene vaccines to prevent and treat hepatocellular carcinoma: review and future prospects. Immunotherapy 2014;6:725-36.

66. Nakagawa H, Mizukoshi E, Kobayashi E, Tamai T, Hamana H, et al. Association between high-avidity T-cell receptors, induced by $\alpha$-fetoprotein-derived peptides, and anti-tumor effects in patients with hepatocellular carcinoma. Gastroenterology 2017;152:1395-406. e10.

67. Liu H, Xu Y, Xiang J, Long L, Green S, et al. Targeting alpha-fetoprotein (AFP)-MHC complex with CAR T-cell therapy for liver cancer. Clin Cancer Res 2017;23:478-88.

68. Parkhurst MR, Yang JC, Langan RC, Dudley ME, Nathan DA, et al. T cells targeting carcinoembryonic antigen can mediate regression of metastatic colorectal cancer but induce severe transient colitis. Mol Ther 2011;19:620-6.

69. Xu Y, Yang Z, Horan LH, Zhang P, Liu L, et al. A novel antibody-TCR (AbTCR) platform combines Fab-based antigen recognition with gamma/delta-TCR signaling to facilitate T-cell cytotoxicity with low cytokine release. Cell Discov 2018;4:62.

70. Lin KR, Deng FW, Jin YB, Chen XP, Pan YM, et al. T cell receptor repertoire profiling predicts the prognosis of HBV-associated hepatocellular carcinoma. Cancer Med 2018;7:3755-62.

71. Qasim W, Brunetto M, Gehring AJ, Xue SA, Schurich A, et al. Immunotherapy of HCC metastases with autologous T cell receptor redirected T cells, targeting HBsAg in a liver transplant patient. J Hepatol 2015;62:486-91.

72. Brechot C, Pourcel C, Louise A, Rain B, Tiollais P. Presence of integrated hepatitis B virus DNA sequences in cellular DNA of human hepatocellular carcinoma. Nature 1980;286:533-5.

73. Gehring AJ, Xue SA, Ho ZZ, Teoh D, Ruedl C, et al. Engineering virus-specific T cells that target HBV infected hepatocytes and hepatocellular carcinoma cell lines. J Hepatol 2011;55:103-10.

74. Tan AT, Yang N, Lee Krishnamoorthy T, Oei V, Chua A, et al. Use of expression profiles of HBV-DNA integrated into genomes of hepatocellular carcinoma cells to select T cells for immunotherapy. Gastroenterology 2019;156:1862-76.e9.

75. Spear TT, Callender GG, Roszkowski JJ, Moxley KM, Simms PE, et al. TCR gene-modified T cells can efficiently treat established hepatitis C-associated hepatocellular carcinoma tumors. Cancer Immunol Immunother 2016;65:293-304.

76. Zhu W, Peng Y, Wang L, Hong Y, Jiang X, et al. Identification of alpha-fetoprotein-specific T-cell receptors for hepatocellular carcinoma immunotherapy. Hepatology 2018;68:574-89.

77. Dargel C, Bassani-Sternberg M, Hasreiter J, Zani F, Bockmann JH, et al. T cells engineered to express a T-cell receptor specific for glypican-3 to recognize and kill hepatoma cells in vitro and in mice. Gastroenterology 2015;149:1042-52.

78. Yin XY, Lu MD, Lai YR, Liang LJ, Huang JF. Prognostic significances of tumor-infiltrating S-100 positive dendritic cells and lymphocytes in patients with hepatocellular carcinoma. Hepatogastroenterology 2003;50:1281-4.

79. Cai XY, Gao Q, Qiu SJ, Ye SL, Wu ZQ, et al. Dendritic cell infiltration and prognosis of human hepatocellular carcinoma. J Cancer Res Clin Oncol 2006;132:293-301.

80. Ladhams A, Schmidt C, Sing G, Butterworth L, Fielding G, et al. Treatment of non-resectable hepatocellular carcinoma with autologous tumor-pulsed dendritic cells. J Gastroenterol Hepatol 2002;17:889-96.

81. Lee WC, Wang HC, Hung CF, Huang PF, Lia CR, et al. Vaccination of advanced hepatocellular carcinoma patients with tumor lysatepulsed dendritic cells: a clinical trial. J Immunother 2005;28:496-504.

82. Palmer DH, Midgley RS, Mirza N, Torr EE, Ahmed F, et al. A phase II study of adoptive immunotherapy using dendritic cells pulsed with tumor lysate in patients with hepatocellular carcinoma. Hepatology 2009;49:124-32.

83. El Ansary M, Mogawer S, Elhamid SA, Alwakil S, Aboelkasem F, et al. Immunotherapy by autologous dendritic cell vaccine in patients with advanced HCC. J Cancer Res Clin Oncol 2013;139:39-48.

84. Kakumu S, Ito S, Ishikawa T, Mita Y, Tagaya T, et al. Decreased function of peripheral blood dendritic cells in patients with hepatocellular carcinoma with hepatitis B and C virus infection. J Gastroenterol Hepatol 2000;15:431-6.

85. Tan XH, Zhu Q, Liu C, Liu XL, Shao XT, et al. Immunization with dendritic cells infected with human AFP adenovirus vector effectively elicits immunity against mouse hepatocellular carcinomas. Zhonghua Zhong Liu Za Zhi 2006;28:13-6. 
86. Yang JY, Cao DY, Xue Y, Yu ZC, Liu WC. Improvement of dendritic-based vaccine efficacy against hepatitis B virus-related hepatocellular carcinoma by two tumor-associated antigen gene-infected dendritic cells. Hum Immunol 2010;71:255-62.

87. Shi M, Qian S, Chen WW, Zhang H, Zhang B, et al. Hepatitis B virus (HBV) antigen-pulsed monocyte-derived dendritic cells from HBVassociated hepatocellular carcinoma patients significantly enhance specific T cell responses in vitro. Clin Exp Immunol 2007;147:277-86.

88. Guo DW, Zhang SY, Hou XZ, Li HW, Jiang XF, et al. Glypican3 in genetically modified human monocyte-derived dendritic cells induced specific cytotoxity against glypican3 overexpressing human hepatocellular carcinoma cells in vitro. Saudi Med J 2008;29:1235-40.

89. Tada F, Abe M, Hirooka M, Ikeda Y, Hiasa Y, et al. Phase I/II study of immunotherapy using tumor antigen-pulsed dendritic cells in patients with hepatocellular carcinoma. Int J Oncol 2012;41:1601-9.

90. Lee JH, Lee Y, Lee M, Heo MK, Song JS, et al. A phase I/IIa study of adjuvant immunotherapy with tumour antigen-pulsed dendritic cells in patients with hepatocellular carcinoma. Br J Cancer 2015;113:1666-76.

91. Ge C, Xing Y, Wang Q, Xiao W, Lu Y, et al. Improved efficacy of therapeutic vaccination with dendritic cells pulsed with tumor cell lysate against hepatocellular carcinoma by introduction of 2 tandem repeats of microbial HSP70 peptide epitope 407-426 and OK-432. Int Immunopharmacol 2011;11:2200-7.

92. Nakamoto Y, Mizukoshi E, Kitahara M, Arihara F, Sakai Y, et al. Prolonged recurrence-free survival following OK432-stimulated dendritic cell transfer into hepatocellular carcinoma during transarterial embolization. Clin Exp Immunol 2011;163:165-77.

93. Maeda Y, Yoshimura K, Matsui H, Shindo Y, Tamesa T, et al. Dendritic cells transfected with heat-shock protein 70 messenger RNA for patients with hepatitis $\mathrm{C}$ virus-related hepatocellular carcinoma: a phase 1 dose escalation clinical trial. Cancer Immunol Immunother 2015;64:1047-56.

94. Chen C, Ma YH, Zhang YT, Zhang F, Zhou N, et al. Effect of dendritic cell-based immunotherapy on hepatocellular carcinoma: a systematic review and meta-analysis. Cytotherapy 2018;20:975-89.

95. Ninomiya T, Akbar SM, Masumoto T, Horiike N, Onji M. Dendritic cells with immature phenotype and defective function in the peripheral blood from patients with hepatocellular carcinoma. J Hepatol 1999;31:323-31.

96. Chen S, Akbar SM, Tanimoto K, Ninomiya T, Iuchi H, et al. Absence of CD83-positive mature and activated dendritic cells at cancer nodules from patients with hepatocellular carcinoma: relevance to hepatocarcinogenesis. Cancer Lett 2000;148:49-57.

97. Iwashita Y, Goto S, Tahara K, Kawano K, Chen CL, et al. Potent stimuli combined with lipopolysaccaride and IFNgamma may improve immunotherapy against $\mathrm{HCC}$ by increasing the maturation and subsequent immune response of the dendritic cells. Cancer Biother Radiopharm 2003;18:1-6.

98. Gonzalez-Carmona MA, Lukacs-Kornek V, Timmerman A, Shabani S, Kornek M, et al. CD40ligand-expressing dendritic cells induce regression of hepatocellular carcinoma by activating innate and acquired immunity in vivo. Hepatology 2008;48:157-68.

99. Chan RC, Pang XW, Wang YD, Chen WF, Xie Y. Transduction of dendritic cells with recombinant adenovirus encoding HCA661 activates autologous cytotoxic T lymphocytes to target hepatoma cells. Br J Cancer 2004;90:1636-43.

100. Peng W, Zhao G, Ma Y, Yu H, Wang X. Dendritic cells transfected with PEG10 recombinant adenovirus elicit anti-tumor immune response in vitro and in vivo. Vaccine 2011;29:3501-6.

101. Vogt A, Sievers E, Lukacs-Kornek V, Decker G, Raskopf E, et al. Improving immunotherapy of hepatocellular carcinoma (HCC) using dendritic cells (DC) engineered to express IL-12 in vivo. Liver Int 2014;34:447-61.

102. Choi YJ, Park SJ, Park YS, Park HS, Yang KM, et al. EpCAM peptide-primed dendritic cell vaccination confers significant anti-tumor immunity in hepatocellular carcinoma cells. PLoS One 2018;13:e190638.

103. Yang X, Zhu H, Hu Z. Dendritic cells transduced with TEM8 recombinant adenovirus prevents hepatocellular carcinoma angiogenesis and inhibits cells growth. Vaccine 2010;28:7130-5.

104. Su H, Li B, Zheng L, Wang H, Zhang L. Immunotherapy based on dendritic cells pulsed with CTPFoxM1 fusion protein protects against the development of hepatocellular carcinoma. Oncotarget 2016;7:48401-11.

105. Zerbini A, Pilli M, Fagnoni F, Pelosi G, Pizzi MG, et al. Increased immunostimulatory activity conferred to antigen-presenting cells by exposure to antigen extract from hepatocellular carcinoma after radiofrequency thermal ablation. J Immunother 2008;31:271-82.

106. Hu Z, Chen J, Zhou S, Yang N, Duan S, et al. Mouse IP-10 gene delivered by folate-modified chitosan nanoparticles and dendritic/tumor cells fusion vaccine effectively inhibit the growth of hepatocellular carcinoma in mice. Theranostics 2017;7:1942-52.

107. Lee JH, Lee JH, Lim YS, Yeon JE, Song TJ, et al. Adjuvant immunotherapy with autologous cytokine-induced killer cells for hepatocellular carcinoma. Gastroenterology 2015;148:1383-91.e6.

108. Jung NC, Lee JH, Choi HJ, Hwang SU, Song JY, et al. Dendritic cell immunotherapy combined with cytokine-induced killer cells effectively suppresses established hepatocellular carcinomas in mice. Immunol Invest 2016;45:553-65.

109. Chen BA, Li M, Sun ZY, Li CP, Gao C, et al. Killing activity in DC and CIK co-culture against hepatocarcinoma cells. Zhongguo Shi Yan Xue Ye Xue Za Zhi 2006;14:543-6.

110. Qiu Y, Xu MB, Yun MM, Wang YZ, Zhang RM, et al. Hepatocellular carcinoma-specific immunotherapy with synthesized alpha1,3galactosyl epitope-pulsed dendritic cells and cytokine-induced killer cells. World J Gastroenterol 2011;17:5260-6.

111. Niu LZ, Li JL, Zeng JY, Mu F, Liao MT, et al. Combination treatment with comprehensive cryoablation and immunotherapy in metastatic hepatocellular cancer. World J Gastroenterol 2013;19:3473-80.

112. Yoon JS, Song BG, Lee JH, Lee HY, Kim SW, et al. Adjuvant cytokine-induced killer cell immunotherapy for hepatocellular carcinoma: a propensity score-matched analysis of real-world data. BMC Cancer 2019;19:523.

113. Lee JH, Lee JH, Lim YS, Yeon JE, Song TJ, et al. Sustained efficacy of adjuvant immunotherapy with cytokine-induced killer cells for hepatocellular carcinoma: an extended 5-year follow-up. Cancer Immunol Immunother 2019;68:23-32.

114. Guo W, Liu L, Wu D. Dendritic cell-cytokine induced killer cell immunotherapy combined with transcatheter arterial chemoembolization 
for hepatocellular carcinoma: safety and efficacy. Nan Fang Yi Ke Da Xue Xue Bao 2014;34:674-8.

115. He G, Zheng C, Huo H, Zhang H, Zhu Z, et al. TACE combined with dendritic cells and cytokine-induced killer cells in the treatment of hepatocellular carcinoma: a meta-analysis. Int Immunopharmacol 2016;40:436-42.

116. Zhou Z, Qin H, Weng L, Ni Y. Clinical efficacy of DC-CIK combined with sorafenib in the treatment of advanced hepatocellular carcinoma. J Buon 2019;24:615-21.

117. Gonzalez-Carmona MA, Marten A, Hoffmann P, Schneider C, Sievers E, et al. Patient-derived dendritic cells transduced with an a-fetoprotein-encoding adenovirus and co-cultured with autologous cytokine-induced lymphocytes induce a specific and strong immune response against hepatocellular carcinoma cells. Liver Int 2006;26:369-79.

118. Yu X, Xia W, Zhang T, Wang H, Xie Y, et al. Enhanced cytotoxicity of IL-24 gene-modified dendritic cells co-cultured with cytokineinduced killer cells to hepatocellular carcinoma cells. Int J Hematol 2010;92:276-82.

119. Wang Y, Wang Y, Mu H, Liu T, Chen X, et al. Enhanced specific antitumor immunity of dendritic cells transduced with the glypican 3 gene and co-cultured with cytokine-induced killer cells against hepatocellular carcinoma cells. Mol Med Rep 2015;11:3361-7. 